\title{
How Anisotropic is our Universe?
}

\author{
Emory. F. Bunn, Pedro Ferreira and Joseph Silk \\ Center for Particle Astrophysics, and Departments of Astronomy and Physics, \\ University of California, Berkeley CA 94720-7304
}

\begin{abstract}
Large-scale cosmic microwave background anisotropies in homogeneous, globally anisotropic cosmologies are investigated. We perform a statistical analysis in which the four-year data from the Cosmic Background Explorer satellite is searched for the specific anisotropy patterns predicted by these models and thereby set definitive upper limits on the amount of shear, $(\sigma / H)_{0}$ and vorticity, $(\omega / H)_{0}$, which are orders of magnitude stronger than previous constraints. We comment on how these results might impact our understanding of primordial global anisotropy.
\end{abstract}

PACS Numbers : 98.80.Cq, 98.70.Vc, 98.80.Hw

Typeset using REVTEX 
Fluctuations in the cosmic microwave background radiation (CMBR) provide us with a clean and unique probe of the structure of our universe on both small and large scales [1]. Current experiments are allowing us to learn about processes in the early universe with unparalleled precision, and the prospect of future terrestrial and satellite missions give us reason to hope for a clear and accurate picture of the universe in the near future. The main working hypothesis in our attempts to understand the universe is that we live in what was originally a homogeneous and isotropic spacetime, the Friedmann-Robertson-Walker (FRW) cosmology. The current belief is that some physical mechanism generated perturbations (either primordial, with inflation, or actively, with defects) that evolved through gravitational collapse to form the structures we see now. The smoothness of the CMBR seems to be consistent with such a picture.

Inflation provides the primary motivation for believing that, at least in an initially homogeneous space-time, any primordial anisotropy has been removed. However, inflation is by no means a generic initial condition for the universe. In the absence of inflation, one is likely to have commenced with global metric perturbations that while eventually seeding structure formation may result in a universe that is only asymptotically Friedmann-Robertson-Walker. Bianchi models provide a generic description of homogeneous anisotropic cosmologies. In the spirit of studying an alternative to inflation, we study below the experimental constraints if our universe is only asymptotically FRW. There are distinct features depending on the overall geometry and homogeneity class of the model [2], and in a pioneering paper, Collins and Hawking used analytical arguments to find upper bounds on the amount of shear $\left(\sigma_{0}\right)$ and vorticity $\left(\omega_{0}\right)$ in the universe today, from the absence of any detected CMBR anisotropy. A detailed numerical analysis of such models [3] used experimental limits on the dipole and quadrupole to refine limits on universal rotation. More recently, Barrow has argued [5] that there is no "isotropy problem" in such cosmological models which are maximally anisotropic (i.e. in which shear and vorticity have decayed only logarithmically since the Planck time): if one assumes an equipartition of energy between all modes of the gravitational interaction at the Planck time then the present amplitude of CMBR fluctuations should be compatible 
with current observational limits.

In this Letter we improve on previous bounds on the total shear in the universe. We use the most recent data from the Differential Microwave Radiometers aboard the Cosmic Background Explorer (COBE) [4] to constrain the allowed parameters of a Bianchi model of type $\mathrm{VII}_{h}$; this model is asymptotically close to an open FRW universe and has the richest anisotropy structure of the models we could consider. As pointed out by Barrow, it is also an example of a homogeneous cosmology where the decay in $\sigma$ from the Planck time to the present is minimal.

One can describe Bianchi cosmologies in terms of the metric

$$
g_{\mu \nu}=-n_{\mu} n_{\nu}+a^{2}[\exp (2 \beta)]_{A B} e_{\mu}^{A} e_{\nu}^{B}
$$

where $n_{\alpha}$ is the normal to spatial hypersurfaces of homogeneity, $a$ is the conformal scale factor, $\beta_{A B}$ is a 3 matrix only dependent on cosmic time, $t$, and $e_{\mu}^{A}$ are invariant covector fields on the surfaces of homogeneity, which obey the commutation relations

$$
e_{\mu ; \nu}^{A}-e_{\nu ; \mu}^{A}=C_{B C}^{A} e_{\mu}^{B} e_{\nu}^{C}
$$

The structure constants $C_{B C}^{A}$ can be used to classify the different models. We shall focus on type $\mathrm{VII}_{h}$ which has structure constants $C_{31}^{2}=C_{21}^{3}=1, C_{21}^{2}=C_{31}^{3}=\sqrt{h}$. It is convenient to define the parameter $x=\sqrt{h / 1-\Omega_{0}}$, which determines the scale on which the principal axes of shear and rotation change orientation. By taking combinations of limits of $\Omega$ and $x$ one can obtain Bianchi I, V and $\mathrm{VII}_{0}$ cosmologies.

We are interested in large-scale anisotropies so it suffices to evaluate the peculiar redshift a photon will feel from the epoch of last scattering (ls) until now (0)

$$
\Delta T_{A}(\hat{\mathbf{r}})=\left(\hat{r}^{i} u_{i}\right)_{0}-\left(\hat{r}^{i} u_{i}\right)_{l s}-\int_{l s}^{0} \hat{r}^{j} \hat{r}^{k} \sigma_{j k} d \tau
$$

where $\hat{\mathbf{r}}=(\cos \theta \sin \phi, \sin \theta \sin \phi, \cos \phi)$ is the direction vector of the incoming null geodesic, $\mathbf{u}$ is the spatial part of the fluid four-velocity vector and to first order, the shear is $\sigma_{i j}=\partial_{\tau} \beta_{i j}$. To evaluate expression (3), one must first of all determine a parameterization of geodesics on this spacetime. This is given by 


$$
\begin{aligned}
\tan \left(\frac{\phi(\tau)}{2}\right) & =\tan \left(\frac{\phi_{0}}{2}\right) \exp \left[-\left(\tau-\tau_{0}\right) \sqrt{h}\right] \\
\theta(\tau) & =\theta_{0}+\left(\tau-\tau_{0}\right) \\
-\frac{1}{\sqrt{h}} \ln \left\{\sin ^{2}\left(\frac{\phi_{0}}{2}\right)\right. & \left.+\cos ^{2}\left(\frac{\phi_{0}}{2}\right) \exp \left[2\left(\tau-\tau_{0}\right) \sqrt{h}\right]\right\}
\end{aligned}
$$

Solving Einstein's equations (and assuming that matter is a pressureless fluid) one can determine $\mathbf{u}$ and $\sigma_{i j}$. A general expression for (3) was determined in [3]:

$$
\begin{aligned}
& \Delta T_{A}(\hat{\mathbf{r}})=\left(\frac{\sigma}{H}\right)_{0} \frac{2 \sqrt{1-\Omega_{o}}}{\Omega_{0}} \\
& \times\left\{\left[\sin \phi_{0} \cos \theta_{0}-\sin \phi_{l s} \cos \theta_{l s}\left(1+z_{l s}\right)\right]\right. \\
& \left.-\int_{\tau_{l s}}^{\tau_{0}} \frac{3 h\left(1-\Omega_{0}\right)}{\Omega_{0}} \sin 2 \phi[\cos (\theta)+\sin (\theta)] \frac{d \tau}{\sinh ^{4}(\sqrt{h} \tau / 2)}\right\}
\end{aligned}
$$

As shown in [2], [3], the "patterns" in such a model are easy to describe: for $\Omega_{0}<1$ and a finite $x$ one will obtain a spiral with approximately $N=2 / \pi x$ complete twists, focused towards the axis of rotation with an angular size of order $1 / \Omega_{0}$. Taking $x \rightarrow \infty$ will leave only a hotspot.

We will approach the problem of constraining the parameters of these Bianchi models in the following way. For fixed values of the parameters $x$ and $\Omega_{0}$, we will attempt to place upper limits on the amplitude of the shear $(\sigma / H)_{0}$ (or equivalently vorticity $(\omega / H)_{0}$ [6]). The statistics problem we face differs substantially from the situation encountered in placing constraints on more standard models. In standard cosmological models, the predicted CMB anisotropy is a realization of an isotropic Gaussian random field, and its statistical properties are therefore entirely characterized by the power spectrum $C_{l}$. In contrast, in the Bianchi models at least part of the CMB fluctuation comes from the large-scale anisotropy of space; this contribution to the anisotropy takes the form of a particular pattern on the sky, and is not described by the statistics of a Gaussian random field. We therefore require different statistical techniques from those used in previous analyses.

Let $\Delta T(\hat{\mathbf{r}})$ be the temperature fluctuation in the direction of the unit vector $\hat{\mathbf{r}}$. We assume that $\Delta T$ is the sum of two contributions: 


$$
\Delta T(\hat{\mathbf{r}})=\Delta T_{A}(\hat{\mathbf{r}})+\Delta T_{I}(\hat{\mathbf{r}}) .
$$

Here $\Delta T_{A}$ is defined in (5) and $\Delta T_{I}$ represents the "isotropic" residual fluctuation caused by variations in the density and gravitational potential. We call $\Delta T_{I}$ "isotropic" because we assume that it is described by the statistics of an isotropic Gaussian random field. That is, if we expand it in spherical harmonics,

$$
\Delta T_{I}(\hat{\mathbf{r}})=\sum_{l, m} a_{l m} Y_{l m}(\hat{\mathbf{r}}),
$$

then the coefficients $a_{l m}$ are independent Gaussian random variables with zero mean and variances given by an angular power spectrum $C_{l}:\left\langle a_{l m} a_{l^{\prime} m^{\prime}}^{*}\right\rangle=C_{l} \delta_{l l^{\prime}} \delta_{m m^{\prime}}$.

One can naively assume that this set of Gaussian perturbations was generated through the amplification of quantum fluctuations, as in the case of inflation (however see [7]) and it is quite conceivable that this initial set of perturbations is strongly biased (or anti-biased) due to the large anisotropy at early times. To change our results considerably, this would have to compensate (anticompensate) late time evolution of the overall anisotropy on many scales, i.e. there would have to be a strong correlation between the primordial quantum generation of perturbations and subsequent classical evolution of the different temperature variables such that they would interfere (destructively or constructively) for many modes of the temperature autocorrelation function, something that we believe to be unlikely. Another possibility is that subsequent evolution of perturbations will be locked in to the specific orientation of the large scale anisotropy, but if we assume that the dominant source of perturbations are scalar and therefore are only sensitive to the overall volume change of the spacetime, than we can discard this hypothesis. This reasoning does not hold if the dominant source of perturbations comes from tensor modes, but we shall not consider this possibility here.

The anisotropic component $\Delta T_{A}$ does not obey Gaussian statistics. Rather, for fixed values of $x, \Omega_{0}$, and $\sigma$, we can compute the exact pattern $A(\hat{\mathbf{r}})$ of the CMB anisotropy. Unfortunately, we do not know the orientation of this pattern on the sky. We can say that

$$
\Delta T_{A}=A(\mathbf{R} \hat{\mathbf{r}}),
$$


where $A$ is the known pattern of anisotropy and $\mathbf{R} \in S O(3)$ is a $3 \times 3$ rotation matrix. The matrix $\mathbf{R}$ can be specified by three Euler angles $(\theta, \psi, \varphi)$, but we have no knowledge a priori of the values of these angles.

We will place constraints on the shear following standard frequentist statistical practice. (This is in contrast to the Bayesian philosophy adopted in much cosmological data analysis.) For any particular model, we define some goodness-of-fit statistic $\eta$ that depends on the data (To give a simple, familiar example, when one is trying to estimate the mean of a set of data, it is customary to choose $\eta$ to be the chi-squared of the data). Having chosen our goodness-of-fit statistic, we compute its value $\eta_{*}$ using the actual data. We then compute the probability $P\left(\eta<\eta_{*}\right)$ that a random data set would have produced a value as good a value as the actual data or better. If this probability is large, then we say that the model is inconsistent with the data. It is customary to choose a significance level $P_{0}$, say 0.95 , and say that a particular model is ruled out at that significance level if $P\left(\eta<\eta_{*}\right)>P_{0}$.

Our choice of $\eta$ is as follows. Each pixel $d_{i}$ of our data set contains contributions from both intrinsic CMB anisotropy and noise:

$$
d_{i}=(\Delta T \star B)\left(\hat{\mathbf{r}}_{i}\right)+N_{i}
$$

here $B$ represents the DMR beam pattern [8], $\hat{\mathbf{r}}_{i}$ is the direction on the sky of the $i$ th data point, and the star denotes a convolution. According to our model, $\Delta T$ includes the two contributions shown in equation (6). $N_{i}$ is the noise in pixel $i$. For the COBE data, it is an excellent approximation to take $N_{i}$ to be Gaussian with zero mean. The correlations between the noise in different pixels are negligible [9], so $\left\langle N_{i} N_{j}\right\rangle=\sigma_{i}^{2} \delta_{i j}$.

Before we give the actual definition of the statistic $\eta$, let us consider a simpler case. Suppose that we knew the geometrical parameters $x, \Omega_{0}$, and $\sigma$, and the rotation matrix $\mathbf{R}$ that defines the orientation of the pattern $A$ on the sky. Then the anisotropic part $\Delta T_{A}$ of the CMB fluctuation would be completely specified, but the isotropic portion $\Delta T_{I}$ and the noise $N_{i}$ would be completely unknown. In this situation, we could define a natural goodness-of-fit statistic in the following way. Let $\Delta_{0}^{2}$ be the noise-weighted mean-square 
value of the data:

$$
\Delta_{0}^{2}=\sum_{i} \frac{d_{i}^{2}}{\sigma_{i}^{2}}
$$

Now let $\Delta_{1}^{2}$ be the mean-square value of the residuals after we have subtracted off the known anisotropic portion:

$$
\Delta_{1}^{2}=\sum_{i} \frac{\left(d_{i}-\left(\Delta T_{A} \star B\right)\left(\hat{\mathbf{r}}_{i}\right)\right)^{2}}{\sigma_{i}^{2}} .
$$

If our model is correct, then we expect $\Delta_{1}^{2}$ to be smaller than $\Delta_{0}^{2}$ : if we have correctly removed a portion of the signal, then the residuals should be smaller, on average. On the other hand, if our model is incorrect, then attempting to remove the anisotropic portion should increase the residuals. The difference between $\Delta_{0}^{2}$ and $\Delta_{1}^{2}$ is therefore a natural choice of goodness-of-fit statistic. In practice, it is more convenient to divide by $\Delta_{0}^{2}$, in order to make the results more weakly dependent on the amplitude of the isotropic cosmological signal $\Delta T_{I}$. We therefore define our statistic to be

$$
\eta_{1}=\frac{\Delta_{0}^{2}-\Delta_{1}^{2}}{\Delta_{0}^{2}}
$$

In fact, of course, we cannot use the statistic (12), because we do not know the parameters necessary to determine $\Delta T_{A}$. In particular, we do not know $\mathbf{R}$ or $\sigma$. (We have chosen to set up the problem as one of constraining $\sigma$ for fixed $x$ and $\Omega_{0}$, so we can assume that we know the latter two parameters.) But we can define a new statistic $\eta$ whose value is the minimum of $\eta_{1}$ over the unknown parameters:

$$
\eta=\min _{\sigma, \theta, \psi, \varphi} \eta_{1}
$$

The statistical task we have set ourselves is simple in principle, although it is somewhat cumbersome computationally. For fixed values of the parameters $x$ and $\Omega_{0}$, we must compute the value $\eta_{*}$ of the statistic (13) for the real data. We must then perform Monte Carlo simulations to determine the theoretical probability distribution of $\eta$ to see how consistent the actual value is with each theoretical model. We must perform these simulations for a 
variety of different values of the shear $\sigma$ in order to see which values of $\sigma$ are consistent with the data.

Each calculation of $\eta$ involves a minimization in a four-dimensional parameter space. Since we need to compute $\eta$ repeatedly in our simulations, it is important to perform this calculation efficiently. We chose to reduce the numbers of pixels in the COBE data set by binning pixels together in groups of four (i.e., working in "pixelization level 5" rather than level 6). Since the anisotropy pattern $\Delta T_{A}$ tends to have power on larger scales than either the noise or the isotropic signal, this binning does not reduce our sensitivity very much.

The task of finding the minimum in equation (13) is not trivial, since the function $\eta_{1}(\sigma, \theta, \psi, \varphi)$ has numerous local minima. We use Powell's method for finding minima, but we have to try multiple starting points in order to be confident that we had found, if not the true minimum, at least a local minimum that was almost as low as the true minimum. We chose to adopt the following procedure. We choose $p$ random points in parameter space and evaluate $\eta_{1}$ at each. Starting from the point that gave the lowest value of $\eta_{1}$, we use Powell's method to find a local minimum. We then repeat this entire procedure $q$ times, and we take the lowest value found to be our statistic $\eta$. After some experimentation, we found that choosing $p=10$ and $q=4$ gave reasonable results. Of course, it is essential to use precisely the same procedure for determining $\eta$ in both the real data and the simulations.

We perform our analysis on the four-year DMR data set 4 . We use the ecliptic pixelization of the data. Before performing any analysis, we average together the two $53 \mathrm{GHz}$ maps and the two $90 \mathrm{GHz}$ maps to make a single sky map. The averaging is performed with weights inversely proportional to the squares of the noise levels, in order to minimize the noise in the average map. In order to reduce Galactic contamination, we excise all pixels that lie within the "custom cut" described by the COBE group [10]; this reduces the number of pixels in the map from 6144 to 3890 . We then remove a best-fit monopole and dipole from the map. As mentioned above, we degrade the map from pixelization level 6 to level 5 , and compute the statistic $\eta$ for a grid of points in the $\Omega_{0}-x$ plane.

In order to convert these $\eta$ values into constraints on the shear, we need to determine 
the probability distribution of $\eta$ via Monte Carlo simulations. We performed simulations on a sample of 10 models in the $\Omega_{0}-x$ plane, using three or four values of $\sigma$ for each model. For each choice of the three parameters $\left(\Omega_{0}, x, \sigma\right)$, we created between 200 and 500 random DMR data sets according to equation (9). For simplicity, we assumed that the isotropic component $\Delta T_{I}$ of the anisotropy was given by a scale-invariant power spectrum $C_{l}^{-1} \propto l(l+1)$, although our final results are not very sensitive to this assumption. [Specifically, if we steepen the power spectrum to an effective $n=1.5$ spectrum (see, e.g., [1] for a definition), the limits in Figure 1 change by $\sim 20 \%$.] We processed each sky map in the same way as the real data to determine a value of $\eta$.

We found that in every case the probability distribution of $\eta$ was slightly skew-positive and had tails that were consistent with exponential distributions. We determined the first three moments of each probability distribution, and found that each distribution was very well approximated by a stretched, offset chi-squared distribution, where the three parameters of the distribution (stretch, offset, and number of degrees of freedom) were chosen to fit the three moments [1]. For points in parameter space where we have not performed simulations, we assume that the probability distribution is also well approximated by a stretched chisquared distribution, and we determine the three parameters of the distribution by smoothly interpolating between the points where we have performed simulations.

Having estimated the probability distribution of $\eta$ for the various theoretical models in this way, we are able to set limits on the shear. For each point in our grid in the $\Omega_{0}-x$ plane, we determine the range of values of $\sigma$ such that $P\left(\eta<\eta_{*}\right)<0.95$. We find that $\sigma=0$ is always allowed at the $95 \%$ confidence level; i.e., we do not detect shear at this level. Figure 1 shows the upper limits we can set on $(\sigma / H)_{0}$ and $(\omega / H)_{0}$ as a function of $\Omega_{0}$ and $x$ : for $\Omega_{0}=1$ universes we find that $(\sigma / H)_{0}<3 \times 10^{-9}$ (or $(\omega / H)_{0}<10^{-6}$ ) for any $x>.05$, while for $\Omega_{0}<1$ the upper bounds are even tighter.

These values are to be compared with the constraints from [3] which are typically one to two orders of magnitude higher and relied entirely on the quadrupole: at that time $Q \simeq 7 \times 10^{-5} K$ compared to $Q \simeq 1-2 \times 10^{-5} K$ from the COBE DMR data [4]. Moreover, 


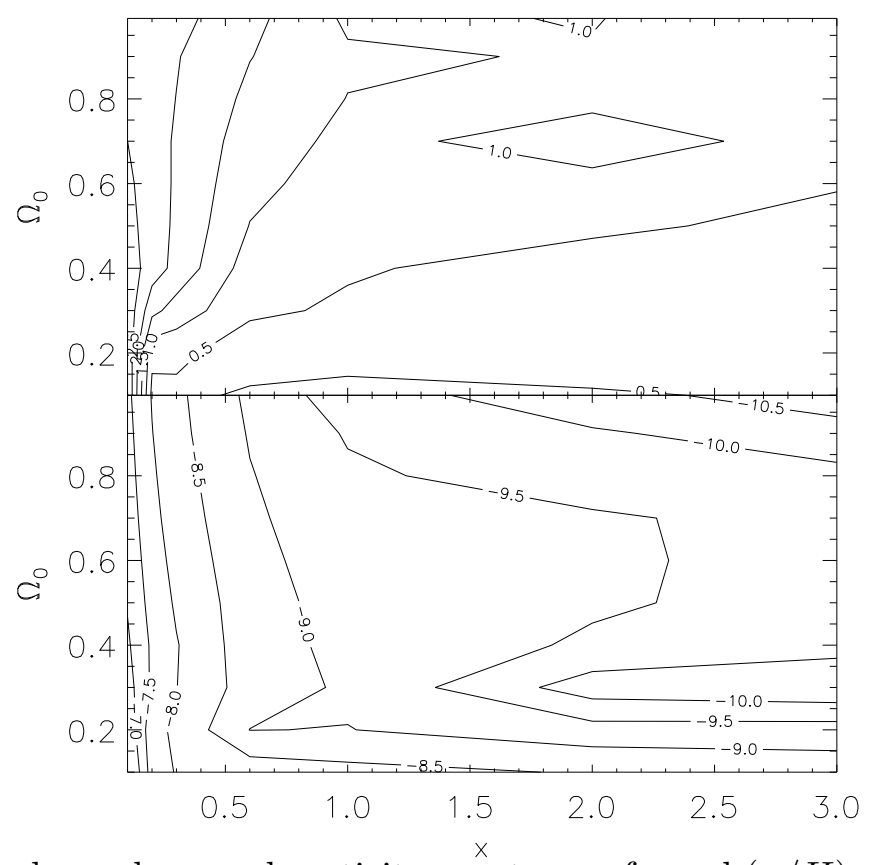

FIG. 1. Upper bounds on shear and vorticity: contours of equal $(\sigma / H)_{0} \times 10^{9}$ and $\log _{10}(\omega / H)_{0}$ are shown in the upper and lower panels respectively for a class of Bianchi $\mathrm{VII}_{h}$ models.

in discarding information from higher moments, their comparison was not sensitive to the small-scale structure present in anisotropic models that is associated either with the spiral pattern (which introduces power on smaller scales as x increases) or with geometrical focusing when $\Omega<1$.

Our tighter constraints rule out the Planck equipartition principle for primordial global shear and vorticity in its most general form [5]. If we consider logarithmic decay of shear through the radiation era due to collisionless stresses, then a rough estimate gives $(\sigma / H)_{P l} \simeq$ $(\sigma / H)_{0}\left(1+z_{\text {curv }}\right)\left(1+z_{e q}\right)^{-1}\left\{1+\ln \left(t_{e q} / t_{P l}\right)\right\}^{-1}$ where $z_{\text {curv }}\left(z_{e q}\right)$ is the redshift of curvature (matter) domination. For the "best" case of $\Omega_{0}=1$ we obtain $(\sigma / H)_{p l} \simeq 10^{-3}-10^{-4}$. Our argument applies to the most general allowed set of globally anisotropic models: generic open or flat homogeneous, anisotropic but asymptotically Friedmann models. While it is possible that for more rapid decay of shear one can allow some relic anisotropy, we conclude that any residual shear or vorticity is constrained to be so small that it is most unlikely to have had a Planck era origin.

ACKNOWLEDGMENTS: We acknowledge conversations with John Barrow, Janna 
Levin, and Martin White. We acknowledge partial support by NASA and DOE. P.F. was supported by the Center for Particle Astrophysics, a NSF Science and Technology Center at U.C. Berkeley, under Cooperative Agreement No. AST 9120005. 


\section{REFERENCES}

[1] M. White, D. Scott and J. Silk, Annu. Rev. Atron. Astrophys. 32 319-370 (1994).

[2] G. F. R. Ellis and M. A. H. MacCallum Commun. Math. Phys. 12 108-141 (1969); S. Hawking M.N.R.A.S 142 129-141 (1969); C. B. Collins and S. W. Hawking M.N.R.A.S. 162 307-320 (1973); A. G. Doroshkevich, V. N. Lukash and I. D. Novikov Sov. Astron. $18554-560(1975)$;

[3] J. D. Barrow, R. Juszkiewicz and D. H. Sonoda M.N.R.A.S 213 917-943 (1985)

[4] C.L. Bennett et al., preprint astro-ph/9601067.

[5] J. D. Barrow Phys. Rev. 51 3113-3116 (1995)

[6] We can relate these two quantities through: $(\omega / H)_{0}=(\sigma / H)_{0}[2(1+h)(1+9 h)]^{1 / 2} /\left(6 x^{2} \Omega_{0}\right)$.

[7] R. M. Wald Phys. Rev. D28 2118-2120 (1983); L. G. Jensen and J. A. Stein-Schabes Phys. Rev. D34 931-933 (1986); M. S. Turner and L. M. Widrow Phys. Rev. Lett. 57 2237-2240 (1986);

[8] E.L. Wright et al. Astrophys. J. 4201 (1994).

[9] C.H. Lineweaver et al. Astrophys. J. 436 452-455 (1995).

[10] Kogut et al., preprint astro-ph/9601060 (1996).

[11] M. Tegmark, E.F. Bunn, and W. Hu Astrophys. J. 434 1-11 (1994). 